\title{
Transient late gestation prenatal hypoxic insult results in functional deficits but not gross neuroanatomic deficits in mice
}

Ana G. Cristancho ${ }^{a, b, c}$, Elyse C. Gadra ${ }^{d}$, Ima M. Samba ${ }^{a}$, Chenying Zhao ${ }^{e, f}$, Minhui Ouyang ${ }^{e, g}$, Sergey

Magnitskye, Hao Huang ${ }^{\mathrm{e}, \mathrm{g}}$, Angela N. Viaene ${ }^{\mathrm{h}}$, Stewart A. Anderson ${ }^{\mathrm{d}, \mathrm{i}}$, and Eric D. Marsh ${ }^{\mathrm{a}, \mathrm{b}, \mathrm{c}}$

${ }^{a}$ Division of Child Neurology, ${ }^{b}$ Department of Pediatrics, Children's Hospital of Philadelphia, Philadelphia, PA, USA

${ }^{\mathrm{C}}$ Department of Neurology, Perelman School of Medicine, University of Pennsylvania, Philadelphia, PA, USA

${ }^{\mathrm{d}}$ Department of Child and Adolescent Psychiatry and Behavioral Services, The Children's Hospital of Philadelphia, Philadelphia, PA, USA

${ }^{\text {e }}$ Radiology Research, Children's Hospital of Philadelphia Research Institute, Philadelphia, PA, USA

${ }^{f}$ Department of Bioengineering, School of Engineering and Applied Science, University of

Pennsylvania, Philadelphia, PA, USA

${ }^{\mathrm{g}}$ Department of Radiology, Perelman School of Medicine, University of Pennsylvania, PA, USA

${ }^{\mathrm{h}}$ Department of Pathology and Laboratory Medicine, Children's Hospital of Philadelphia,

Philadelphia, PA, USA and Perelman School of Medicine, University of Pennsylvania, Philadelphia, PA, USA

'Department of Psychiatry, Perelman School of Medicine, University of Pennsylvania, Philadelphia, PA, USA

Short Title: Deficits from transient prenatal hypoxia

Corresponding Author:

Ana G. Cristancho, MD, PhD

Abramson Research Center, Rm. 516g

Children's Hospital of Philadelphia,

3615 Civic Center Blvd.

Philadelphia, PA. 19104, USA

Tel: 267-426-6892

Email: cristanchoa@chop.edu

Number of Tables: 0

Number of Figures: 7

Word count: 6997

Keywords: prenatal hypoxia, animal model, behavior, imaging 


\section{Abstract}

2 Intrauterine hypoxia is a common cause of brain injury in children with a wide spectrum of long-term 3 neurodevelopmental sequela. Even an insult that does not result in significant neuroanatomical $4 \quad$ injury in perinatal brain imaging can lead to lifelong disabilities. Commonly used postnatal HIE 5 models are not able to directly study the effects of pregnancy risk factors that contribute to outcomes of hypoxia in children. Large animal models suggest that transient prenatal hypoxia alone is sufficient to lead to significant functional impairment to the developing brain but published rodent prenatal hypoxia models are complex (requiring prolonged [days] hypoxic exposure or difficult surgical procedures) and can be difficult to replicate. Thus, to further understand the mechanisms underlying hypoxic injury seen in children affected by mild intrauterine hypoxia, murine models that are simple to reproduce and phenocopy the lack of neuroanatomic injury but have significant functional deficits are needed. Here we characterized the effect of late gestation (embryonic day 17.5) transient prenatal hypoxia on long-term anatomical and neurodevelopmental outcomes. Late gestation transient prenatal hypoxia increased hypoxia-inducible factor 1 alpha protein levels (a marker of hypoxic exposure) in the fetal brain but did not result in any difference in gestational age at birth, litter size at birth, or pup survival. In addition, there were no differences in fetal brain cell death or long-term changes in gray or white matter between offspring after normoxia and hypoxia. However, there were several long-term functional consequences from prenatal hypoxia, including sex-dichotomous changes. Both males and females had abnormalities in repetitive behaviors, hindlimb strength, and decreased seizure threshold. Males demonstrated increased anxiety. Females had deficits in social interaction. Hypoxia did not result in motor or visual learning deficits. This work demonstrates that transient late gestation prenatal hypoxia is a simple, clinically relevant paradigm for studying putative environmental and genetic modulators of the long-term effects of transient hypoxia on the developing brain. 


\section{Introduction}

Neonatal hypoxic ischemic encephalopathy (HIE) is due to an intrapartum loss of oxygen and nutrients that impacts millions of births and results in lives lived with disability, including neurodevelopmental disabilities (NDDs), such as autism, and epilepsy [1, 2]. Children who are initially classified as having mild HIE, defined as minimal evidence of perinatal cell death by imaging and relatively normal neonatal physical exam, can still have adverse outcomes [3-8]. These children do not qualify for therapeutic hypothermia, the only specific intervention for moderate-severe HIE [9]. The incidence of HIE is disproportionately higher in children born in areas with limited prenatal care or access to therapies [10] so there is an urgent need for novel, widely available interventions.

To understand HIE it is important to consider that in utero hypoxic injury differs from postnatal injury. The in utero environment is relatively hypoxic at baseline [11]. The required oxygen tension during fetal development is tightly regulated as evidenced by brain injury either from early exposure to relative hyperoxia in premature children or due to in utero hypoxia $[12,13]$. While HIE is primarily an in utero injury, the most commonly used rodent model is a unilateral carotid ligation followed by hypoxia at day of life 7-10 (Vannucci Model) [14]. This model has provided many insights [15, 16], in part, because neuroanatomically the rodent brain is considered human "term" equivalent at postnatal day 7-10 [17]. However, injury in the Vannucci model is has significant cell death, which is most consistent with severe injury, and injury is very focal, which is most consistent with perinatal stroke $[14,18]$. Postnatal models also cannot directly study the important contributions of the maternalplacental unit to HIE severity $[19,20]$. Lastly, functional networks do not correlate with neuroanatomic maturation in rodents [21]; mice do not have the respiratory or feeding dyscoordination at birth that is characteristic of children born before 34 weeks gestation [22], and they can walk by postnatal day 10 , which is a developmental milestone acquired in infants at about a 12 months [21, 23]. Therefore, it is important to develop prenatal hypoxia models to understand key aspects of the pathophysiology of HIE as they affect brain development.

Mouse models of prenatal hypoxia exist, but they largely study the effects of chronic hypoxia by exposing dams to mild hypoxia ( 10\% inspired oxygen) throughout gestation $[24,25]$ or are technically challenging (late gestation uterine artery ligation) [26]. There have been a few studies of transient prenatal hypoxia in rodents $[27,28]$, but most of our understanding of brief, mild insults is from large animal studies. In sheep, transient prenatal hypoxia leads to long-term structural and functional injury despite less cell death than hypoxia with ischemia, including deficits in neuron dendritic complexity and action potential propagation [29, 30].

To complement existing models and determine if transient hypoxia has similar findings as 
seen in large animal studies, here we characterize a simplified model of transient prenatal hypoxia. In particular, we focus on early and late neuroanatomic injury using clinically anaolgous pathology and neuroimaging measures as well as wide battery of behavioral studies to determine whether this model correlates to mild hypoxic injury seen in children.

\section{Materials and Methods}

\section{Animals}

Male and female C57BI/6 mice were purchased from Charles River Laboratories (Wilmington, MA). Timed matings were used to ensure consistent timing of prenatal hypoxia exposures. Mice were maintained at the Children's Hospital of Philadelphia Animal Facility at a 12-hour light, 12-hour dark cycle (0615-1815 h) and ad libitum access to water and diet mouse food 5015 (LabDiet, St. Louis, MO). Offspring exposed to prenatal normoxia or hypoxia were periodically weighed by experimenters blinded to exposure. Animals were group housed for postnatal experiments. Male and female gonadectomized CD1 mice for Social Interaction were acquired from Charles River Laboratories. The Institutional Animal Care and Use Committee of the Children's Hospital of Philadelphia approved all experiments.

\section{Prenatal hypoxia}

Pregnant females were placed in a controlled oxygen chamber (BioSpherix Ltd., Parish, NY) at embryonic day 17.5 (E17.5). Mice were acclimated to the chamber for 1-2 minutes at 21\% oxygen. For hypoxic exposures, oxygen concentration was decreased to 5\% oxygen over 30 minutes and maintained at $5 \%$ oxygen for duration of experiment $(2,4,6$, or 8 hours). For normoxic control exposures, pregnant mice were placed in the same controlled oxygen chamber for 8 hours. Dams in both groups were provided free access to food and hydrogel for hydration throughout the time in chamber. For locomotion, pregnant mice (normoxia $n=4$, hypoxia $n=4$ ) were videotaped during first 90 minutes in the chamber and cumulative distance the mouse moved in chamber was quantified with ANY-maze software (Stoelting Co., Wood Dale, IL, USA). Except for protein and RNA studies, after hypoxia, pregnant dams were monitored for recovery and demonstrated normal locomotion within 10 minutes of end of exposure. All survival studies were performed after 8 hours of prenatal hypoxia. Pregnant dams from normoxia and hypoxia were assessed twice per day until delivery to determine gestational age at birth and number of pups per litter at birth (normoxia $n=19$, hypoxia $n=28$. 
92

\section{$R N A$ isolation and quantitative $P C R$}

Messenger RNA (mRNA) was isolated from flash frozen samples using RNeasy Lipid Tissue Mini Kit (QIAGEN, Venlo, Netherlands). RNA was isolated with DNAse to avoid contamination. Individual samples shown were isolated from $n=11-12$ fetal brains/condition in 3 separate litters/condition. RNA was converted to cDNA with High-Capacity cDNA Reverse Transcription Kit (Applied Biosystems, Foster City, CA). Quantitative PCR on Quant Studio 12K Flex (Applied Biosystems, Foster City, CA) was used to quantitate mRNA levels with Taqman of $18 \mathrm{~s}$ as a housekeeping gene (Mm03928990_g1, Thermo Fisher Scientific, Waltham, MA) and the HIF1 $\alpha$ target gene, Vegfa (Mm00437306_m1, Thermo Fisher Scientific, Waltham, MA). Vegfa levels were normalized to $18 \mathrm{~S}$ levels in each sample. To normalize between different plates, average of the normoxic condition, were standardized to 1 and all other samples on that plate were multiplied by the sample standardization factor.

\section{Maternal nestlet}

Ability for pregnant dams to form a nest after normoxia or hypoxia was used as a proxy for the ability to form a nurturing environment. A 5-point scale was utilized as previously described, with a score of 5 being an intact nest [31]. In brief, after 8 hours in oxygen chamber in normoxia or hypoxia, pregnant dams were placed in new cage with intact cotton squares and no other environment enrichment cage. They were placed in standard holding room overnight. The following morning an experimenter obtained an overhead picture of the cage with minimal disruption. A blinded experimenter scored the pictures.

\section{Histology}

Fetal brains were harvested 24 hours after exposure and placed in $4 \%$ paraformaldehyde. Fixed brains were processed and paraffin-embedded and then sectioned in the coronal plane by the CHOP Pathology Core into $5 \mu \mathrm{M}$ unstained slides. Every fifth slide was stained with hematoxylin and eosin and examined to identify brain regions of interest. Apoptotic nuclei in cortex, basal ganglia, and white matter were counted by a neuropathologist (A.N.V.) who was blinded to condition.

\section{Ex vivo MRI}



delineate neuroanatomy [32-34]. Here we used high resolution DTI $(0.1 \times 0.1 \times 0.1 \mathrm{~mm})$ with MR scanner of high magnetic strength to examine the morphological and microstructural changes in mice with hypoxia. MRIs for 7 normoxic animals and 6 hypoxic animals were analyzed. diameter of $15 \mathrm{~mm}$ was used as RF transmitter and receiver. A 3D high-resolution, multi-shot echo planar imaging (EPI) sequence with eight shots was used to acquire diffusion-weighted images (DWIs). The parameters for DWIs were as follows: field of view $=25.6 \times 12.8 \times 10.0 \mathrm{~mm}$; voxel size $=0.1 \times 0.1 \times 0.1 \mathrm{~mm}$; echo time $=26 \mathrm{~ms}$; repetition time $=1250 \mathrm{~ms} ; 6$ or 30 independent diffusionweighted directions with $\mathrm{a} b$ value of $1500 \mathrm{~s} / \mathrm{mm}^{2}$ and five additional images without diffusion gradients; two averages.

Diffusion tensor fitting diagonalization of tensor to obtain three eigenvalues $\left(\lambda \_(1-3)\right)$ and eigenvectors ( $\left.v \_(1-3)\right)$, mean diffusivity (MD) was calculated as the mean of three eigenvalues ( $\left.\lambda \_(1-3)\right)$. Fractional anisotropy (FA) was calculated as follows. referencing known stereotactic reference atlas [36] in ROIEditor (http://www.MRIstudio.org). For genu and splenium of the corpus callosum, the ROIs of consistent size were drawn on seven slices around mid-sagittal slice of the brain. The volume of the ventricles was calculated as the number of voxels in the ROI times the voxel size. The thickness of anterior cingulate cortex was measured in the mid-sagittal region. Volume and thickness measurements were calculated in Matlab (Mathworks, Inc., Natick, MA, USA). 
Behavior studies following published protocols were performed sequentially starting at 3 months old in the order listed below for all animals [37-43]. Any modifications are listed below. Three separate cohorts consisting of a total of 5 normoxic litters and 4 hypoxic litters were used. Composition of experimental groups consisted of normoxic male $=16$, hypoxic male $=10$, normoxic female $=22$, hypoxic female $=13$.

All testing except for grip strength was performed between $0700 \mathrm{~h}$ to $1300 \mathrm{~h}$. Grip strength was performed from $1400 \mathrm{~h}$ to $1600 \mathrm{~h}$. All animals were given at least 1 day and up to 7 days of rest between behavior paradigms to minimize carrying effect between studies. Weight was monitored before each test to ensure there was weight loss consistent with poor health or significant fatigue between experimental days. No mice were removed from these experiments for outliers. Animals were not acclimated to handling before Elevated zero maze or Open field, but were subsequently handled daily between experimental days for 5 minutes per cage. Mice were acclimated to experimental room at least 30 minutes prior to protocol. Equipment apparatus was cleaned with cleaning wipes between mice (PDI Sani-Cloth Plus germicidal disposable cloth). ANY-maze software (Stoelting Co., Wood Dale, IL, USA) was used to video-record and score Elevated zero maze, Open field, Social interaction, and Morris water maze.

Elevated zero maze

Elevated zero maze was modified from previous description [43]. In brief, annular $60 \mathrm{~cm}$ diameter beige apparatus was elevated $50 \mathrm{~cm}$ above the ground, with care to ensure it was level. Two opposing quadrants were "open" and the other two opposing quadrants were "closed" with 16 $\mathrm{cm}$ opaque walls but no cover. Experimenter was hidden from the apparatus by black drape surrounding the apparatus. Illumination was provided overhead with open arms approximately 150200 lumens/m2 and closed arms at 50 lumens/m2. Animals were placed in the center of a closed arm and allowed to roam the apparatus freely for 5 minutes. In addition to time in open arm, head entries into the open arms and time freezing (immobile at least $1 \mathrm{sec}$ ) were detected by ANY-Maze. 
184

lumens/m2 range. Experimenter was visually separated from animals in apparatus by black drape. Each mouse was placed in the center of the box and allowed to roam freely in the box for 15 min. ANY-maze scored a mouse to be in the periphery if it was within $13 \mathrm{~cm}$ of the wall of the box and otherwise they were considered to be in the center of the box. The distance traveled during the test in the entire apparatus was also measured.

\section{Marble burying}

Marble burying was modified from previous studies [37, 39]. A white $41.9 \mathrm{~cm} \times 33.7 \mathrm{~cm} \mathrm{x}$ $22.5 \mathrm{~cm}$ box was filled with $5 \mathrm{~cm}$ of fresh bedding. Twenty-four marbles were arranged in a $4 \times 6$ grid $5 \mathrm{~cm}$ apart. Up to 6 mice were tested at the same time so further barriers were placed between the boxes. Illumination was provided by overhead lights with range of 130-150 lumens/m2 at each box. Each mouse was placed in the center of the box for 30 minutes and allowed to roam freely. Pictures were taken before animals were placed of each chamber and then at the end of the 30 minutes. Two blinded reviewers examined images to determine whether marbles were buried greater than $2 / 3 \mathrm{rd}$ into the bedding. Average from the two reviewers of marbles buried for each mouse is reported.

\section{Short term nestlet}

Short term nestlet experiment was modified from previous study [39]. Animals were placed in a clean cage with $1 \mathrm{~cm}$ of fresh bedding. A precut nestlet with all rough edges removed was weighed and placed in the center of the cage. Each mouse was placed in the center of the chamber away from the experimenter and cage lid was placed on top. The mouse was allowed to roam freely in the chamber for 30 minutes. As multiple animals were in the cages at the same time, barriers were placed to visually separate the animals. At the end of the 30 minutes, the animals were removed. Nestlets were removed from the cage and allowed to dry overnight. A blinded experimenter handled all the neslets and weighed them after $>24$ hours of drying. Change in nestlet weight was calculated by subtracting from the pre-experiment nestlet weight.

\section{Rotarod}

Mice were tested on a Rotarod from Ugo Basile (Model 47650, Comerio, Italy) as previously published [41]. The mice were tested in groups, 5 at a time for $5 \mathrm{~min}$ trials with 3 trials per day for 4 days. The Rotarod was used in acceleration mode. The first 2 days, speed increased from $4 \mathrm{rpm}$ up to 
$40 \mathrm{rpm}$. The last 2 days, speed increased from $8 \mathrm{rpm}$ to $80 \mathrm{rpm}$. The speed at falling, grappling the rod without walking for 4 consecutive cycles, or end of 5 minutes for each mouse was recorded as the end of each trial. Between trials the mice were placed back into individual containers and at the end of the last trial placed back in home cages. Intertrial time was 15-20 minutes.

\section{Social interaction}

Social interaction and choice were tested as per previously described [42]. White three chamber apparatus was filled with 1-2 cm of fresh bedding. The experiment was performed in red light without fluorescence to resemble night time environment when mice are most likely to interact. For habituation stage, the experimental mouse was placed for $10 \mathrm{~min}$ in apparatus with an empty clear tube with holes was placed in the center of the left and right chambers. The experimental mouse was then placed in a temporary holding chamber prior to the next stage. For the novel mouse/object stage, a sex matched gonadectomized mouse was placed in the tube on the left. In the tube on the right, a novel object was placed. The experimental animal was then returned to the apparatus for $5 \mathrm{~min}$. In addition to time in chamber, animals were considering to be "sniffing" the tube if the head was within $2 \mathrm{~cm}$ of the tube by ANY-maze.

\section{Grip strength}

The grip strength of the mice was tested using a grip strength meter (Model 080312-3 Columbus Instruments, Columbus, $\mathrm{OH}, \mathrm{USA}$ ) as previously published in the Marsh lab [42]. In brief, each mouse was tested in 6 consecutive trials; 3 were for forelimbs, and 3 for hindlimbs. Each trial was recorded, and the average for each mouse for forelimb and hindlimb trials is reported.

\section{Morris water maze}

Morris water maze was performed in a $128 \mathrm{~cm}$ round plastic tube filled with room temperature water (approximately $21^{\circ} \mathrm{C}$ ) as previously published [38, 42]. A platform was submerged $0.5 \mathrm{~cm}$ below water surface. Non-toxic white tempera paint was used to opacify the water so the platform could not be seen from the ledge. Testing was performed in 3 phases: visual acuity trials, place trials, and probe testing. For both trial phases, in each trial a mouse was allowed to swim for up to $60 \mathrm{sec}$ to find the platform or was led there at the end of the $60 \mathrm{sec}$. Once on the platform, the mouse was maintained on the platform for 15 seconds prior to being dried and placed under a heat 
lamp for the following trial if having difficulty with drying its coat. There were 4 trials per day. The visual acuity of each mouse was tested for 2 days by placing a flag that could be seen above the platform. For each trial, the platform with flag and the location of the mouse was placed in was changed. A mouse was considered to have normal vision if by the 2 nd day it was able to find the platform at least $50 \%$ of the trials before 60 seconds. All mice were deemed to have normal vision. Place trials were then performed for 5 days. Flag was removed from platform and the platform was completely submerged in the southwest quadrant. Visual cues were placed in the north, south, east, and west markers of the tub. For each trial, a mouse was placed in a different location of the tub. Time to platform and path efficiency were measured in ANY-Maze and average of these measures for all the trials for each day was reported. For probe testing, platform was removed from the pool. One hour after last place trial, each mouse was placed in the northeast quadrant and allowed to swim freely for 60 seconds to assess short term memory. Time spent swimming the in southwest quadrant, where the platform had been located, was measured. Twenty-four hours after the last place trial, probe test was repeated to assess long-term memory.

260

Flurothyl seizure threshold

Flurothyl (bis-2,2,2-trifluoroethyl ether, Sigma, St. Louis, MO) testing was modified from procedure previously described $[44,45]$. In brief, 5 to 6 month old mice were place on a platform in a 1.7 L rubber sealed glass chamber (Ikea, Älmhult, Sweden) containing a small amount of the carbon dioxide scavenger, soda lime (Sigma). Flurothyl was infused with syringe pump at a rate of $6 \mathrm{~mL} / \mathrm{hour}$ until the first generalized tonic-clonic seizure (GTC) was observed. The experimenter was blinded to condition during the experiments. Animals were videotaped throughout the experiment. Two blinded scorers used a modified Racine scale to define a generalized tonic-clonic seizure [16].

Statistical analysis

All animal experiments were performed with experimenter blinded to exposure condition. The majority of the data is presented using truncated violin plots with individual mice plotted superimposed on top to display frequency distribution (GraphPad, San Diego, CA). Data are grouped by hypoxia and sex. Markings on violin plots are as follows: center dashed line is the median value and lighter lines demonstrate quartiles. 
compare normoxia and hypoxia maternal nestlet shredding, gestational age, litter size, and MRI studies. Log-rank test was utilized for survival. For RNA, One-Way Nested ANOVA was used in Graphpad. For all other studies, R was used for statistics. Mixed models with generalized estimating equations in geepack package in $\mathrm{R}$ was utilized for majority of comparisons [46]. Linear mixed-effects models in Ime4 package in $\mathrm{R}$ was used for experiments requiring analysis of daily repeated measures (weights, Rotarod, Morris Water Maze) [47]. Cohort was used as a random effect. Independent variables for all models included prenatal hypoxia exposure, sex, birth gestational age, and litter size at birth. Statistical significance displayed for hypoxia, sex, and interaction between hypoxia and sex as hypoxia has been shown to have sex-dichotomous effects [48, 49]. Statistical significance was set at $* p<0.05, * * p<0.01$, and $* * * p<0.001$. A Benjamini-Hochberg correction was used for all $p$-values from mixed model analyses. Statistical subanalysis for differences for hypoxia based on sex were only performed if hypoxia or interaction between sex and hypoxia had an adjusted $p<0.15$ to determine if there were more subtle effects unique to each sex or by condition based on the day. Shapiro-Wilk test was used to determine the normality of the distrubution within each test. If the distribution was normal, the mean and standard deviation are reported. If the distribution was not normal, the median and interquartile range (IQR) is reported.

\section{Results}

Prenatal hypoxia induces the canonical hypoxic response in the fetal brain

First, we performed a time course to determine the duration of prenatal hypoxia that was required to induce a reproducible hypoxic molecular response in the fetal brain. The transcription factor hypoxia inducible factor 1 alpha (Hif1 $\alpha$ ) and its canonical target, vascular endothelial growth factor A (Vegfa), are established molecular markers of the hypoxic response [50]. Hif1 $\alpha$ protein is stabilized by the intrinsically hypoxic in utero environment [51] but it can be induced further by prenatal hypoxia (Fig. 1A \& 1B). Vegfa mRNA levels also increased with prenatal hypoxia (Fig. 1C). While the peak of increase for both protein and mRNA was at 4 hours of hypoxia, there was decreased variability in Vegfa after 8 hours of hypoxia. Thus, 8 hours of hypoxia was used for all further experiments.

Maternal and offspring survival and health were unaffected by prenatal hypoxia Pregnant mice demonstrated a decrease in locomotor activity during hypoxic exposure once oxygen was less than $10 \% \mathrm{FiO}$, approximately 20 minutes after start of the hypoxia protocol (Fig. 
2A). They survived 8 hours of hypoxia and rapidly recovered during brief observation. Given pregnant mice locomotion was affected by hypoxia, we tested whether their ability to rear offspring after hypoxic exposure may be grossly altered. After normoxia or hypoxia, we placed the pregnant mice in a new clean cage with an intact nestlet square and compared the difference in making a nest as a proxy for creating a nurturing environment. Sixteen hours after exposure, almost all mice of both conditions made normal nests (Fig. 2B).

Offspring health after normoxia and hypoxia was assessed by litter size at birth, birth gestational age, survival, and weight. There was no statistical difference in litter size at birth (Fig. 2C) or birth gestational age (Fig. 2D). In addition, there was no difference in survival of mice through the first 90 days of life (Fig. 2E). Together these results suggest that maternal dams can tolerate and appropriately rear offspring after prenatal hypoxia and that there is a not a severe, life threatening phenotype from prenatal hypoxia on the offspring.

\section{Prenatal hypoxia does not increase cell death in the fetal brain}

In the Vannucci model there has been efforts to determine the kinetics of apoptosis after injury where apoptosis peaks at 24-48 hours after injury in the co [52]. Based on this work, we isolated the fetal brain 24 hours after exposure to hypoxia and normoxia (at E18.5), prior to being born to avoid any confounding effects from birth. A neuropathologist (ANV) blinded to the exposure condition then counted apoptotic nuclei (Fig $\mathbf{3 A}$ ) in the cortex, basal ganglia, and white matter, three regions that can have significant cell death in children with acute perinatal hypoxic injury [15]. There was no increase in apoptotic nuclei in any region of the brain analyzed (Fig. 3B-3D).

\section{Prenatal hypoxia does not lead to long-term neuroanatomical deficits}

To determine if there were long-term neuroanatomic deficits, we performed ex vivo MRI in adult animals that were exposed to normoxia or hypoxia prenatally. Lateral ventricle volume was measured and demonstrated a single significantly affected outlier in hypoxic animals (Fig. 4A), but overall, there was no significant increase in lateral ventricle size (Fig. 4B) or differences in cortical thickness of the anterior cingulate (Fig. 4C \& D). Similarly, DTI derived FA measurement of the corpus callosum, an index of the extent of fiber alignment and myelination, demonstrated no difference between groups in white matter microstructure of the genu or splenium of the corpus callosum (Fig.

\section{C, E \& F).}

Prenatal hypoxia leads to multiple behavior deficits in adult animals Multiple behavioral domains were tested to determine if prenatal hypoxia leads to long-term 
deficits that are consistent with differences seen in children with prenatal hypoxic injury. Results could be divided into three categories - deficits in males and females, sex-dichotomous deficits, and no deficits. Children with moderate to severe HIE have an increased risk of developing epilepsy after the acute period $[53,4]$. While we did not observe any spontaneous seizures or see sudden death that would be consistent with uncontrolled epilepsy, we sought to determine if hypoxic animals had a decrease in seizure threshold through flurothyl seizure threshold testing [44]. We determined that hypoxic mice had a decreased seizure threshold (mean/SD normoxic male $=229.3 \mathrm{~s}+/-53.5$, hypoxia male $=200.0 s+/-30.3$, normoxic female $=211.8 s+/-47.9$, hypoxic female $=182.6 s+/-27.7$ ) (Fig.

5A). This data suggest that prenatal hypoxia may predispose animals to subtle network deficits consistent with decreased seizure threshold.

We performed grip strength to determine whether hypoxic mice had long-term motor deficits [54]. There was a difference at baseline between male and female animals, but there was no decrease in forelimb grip strength due to prenatal hypoxia (mean/SD normoxic male $=0.044 \mathrm{~kg}$ of force $+/-0.016$, hypoxic male $=0.045 \mathrm{~kg}$ of force $+/-0.013$, normoxic female $=0.037 \mathrm{~kg}$ of force $+/-$ 0.012, hypoxic female $=0.039 \mathrm{~kg}$ of force $+/-0.009$ ) (Fig. 5B). By contrast, hindlimbs did demonstrate a difference between normoxic and hypoxic animals (mean/SD normoxic male $=0.020 \mathrm{~kg}$ of force $+/$ 0.006 , hypoxic male $=0.020 \mathrm{~kg}$ of force $+/-0.007$, normoxic female $=0.026 \mathrm{~kg}$ of force $+/-0.009$, hypoxic female $=0.021 \mathrm{~kg}$ of force $+/-0.008$ ) (Fig. 5C), suggesting mild strength impairment after prenatal hypoxia.

We also determined if there were any differences in compulsive and repetitive behaviors [37, 39]. Increased marble burying has been associated with an obsessive compulsive related phenotype [39], but decrease in marble burying has been seen in genetic models of autism, suggesting decrease in this typical behavior is also pathogenic $[55,56]$. Hypoxic animals, particularly females, had a decrease in marbles buried (mean/SD normoxic male $=10.34 n+/-5.99$, hypoxic male $=6.30 n+/$ 5.32, normoxic female $=9.55 n+/-4.45$, hypoxic female $=3.85 n+/-2.48)$ (Fig. 5D). Consistent with decreased repetitive behaviors in Marble burying, hypoxic mice demonstrated significant decrease in a short-term nestlet shredding study, but in this assay the deficit was more prominent in males (median/IQR normoxic male $=-0.05 \mathrm{~g} / 0.20$, hypoxic male $=-0.10 \mathrm{~g} / 0.20$, normoxic female $=-0.10$ $\mathrm{g} / 0.38 .$, hypoxic female $=-0.1 \mathrm{~g}+/-0.25$ ) (Fig. 5E).

\section{Prenatal hypoxia leads to some sex-dichotomous behavior differences in anxiety and social} interaction

Anxiety seemed more prevalent in hypoxic males using two different assays. Elevated zero maze is considered a strong anxiogenic stimulus for animals, where increased anxiety is associated 
with decreased time in the open arms of the apparatus [57]. Hypoxic males spent less time in the open arms than normoxic males and there was no effect on females (mean/SD normoxic male $=$ $144.7 \mathrm{~s}+/-51.8$, hypoxia male $=112.0 \mathrm{~s}+/-33.9$, normoxic female $=139.0 \mathrm{~s}+/-53.7$, hypoxic female $=144.9 \mathrm{~s}+/-40.8$ ) (Fig. 6A). To determine if this phenotype was confirmed, we performed Open field testing, another well-established assay for anxiety where increased anxiety is associated with decrease time in the center of the apparatus [57]. Here we observed that hypoxic males spent less time in the center of the field than normoxic males (median/IQR normoxic male $=151.8 \mathrm{~s} / 128.8$, hypoxic male $=127.6 \mathrm{~s} / 105.3)$ (Fig 6B). Notably, females spend less time than males in the center in general and there was no added affect by hypoxia (normoxic female $=86.3 \mathrm{~s} / 69.0$. , hypoxic female $=$ $84.5 \mathrm{~s} / 104.7)$. These differences in Open field testing were not due to differences in total distance travelled $(\mathrm{mean} / \mathrm{SD}$ normoxic male $=36.9 \mathrm{~m}+/-11.5$, hypoxia male $=36.6 \mathrm{~m}+/-10.3$, normoxic female $=40.8 \mathrm{~m}+/-12.2$, hypoxic female $=39.4 \mathrm{~m}+/-13.1, p=0.60$ between normoxia and hypoxia). Hypoxic insults can be associated with social deficits like autism [58], so we performed three chamber social interaction testing. In the initial stage of this test, we demonstrated that mice did not have any preference for the left or right side of the chamber (Fig. 6C \& D). In the second stage, a novel mouse was placed in a cup in the left side of the chamber and a novel object was placed on the right side of the chamber. As expected, normoxic males and females demonstrated a preference for the novel mouse compared to the novel object (Fig. 6E \& F). Hypoxic males demonstrated a similar increase in preference for the novel mouse (Fig. 6E). However, hypoxic females did not have as strong of a preference for the novel mouse (Fig. 6F), suggesting a decrease in social interaction only in female animals.

\section{Prenatal hypoxia does not lead to deficits in learning or memory}

To determine whether prenatal hypoxia led to more significant deficits in learning or memory, we tested motor learning and visual spatial learning and memory. In Rotarod testing for motor learning and coordination [41], there was no difference between hypoxia and normoxia (Fig. 7A).

Morris water maze was used to test for deficits in learning and memory [38]. Place trials demonstrated there was no difference between normoxic and hypoxic mice in time to platform (Fig. 7B). Probe testing in Morris water maze was used to determine if there were any differences in memory. Prenatal hypoxia did not predispose mice of either sex to short or longer term memory deficits (Fig. 7C \& D). Together, this data suggests that this prenatal hypoxia model does not by itself predispose animals to significant cognitive deficits. 


\section{Discussion/Conclusion}

413

414

415

416

417

418

419

420

421

422

423

424

425

426

427

428

429

430

431

432

433

434

435

436

437

438

439

440

441

442

443

444

Here we have demonstrated that a simple prenatal hypoxia model is able to recapitulate some of the features seen in children with mild HIE. Specifically, after prenatal hypoxia, animals have evidence of molecular induction of hypoxic response, minimal differences in early markers of injury and long-term gross neuroanatomic injury, but evidence of long-term functional deficits. Some if these deficits are analogous to neurodevelopmental disabilities seen in children after HIE, including in deficits in anxiety [59], motor function [60], and susceptibility to seizures [4, 61]. The functional deficits in the absence of long-term structural deficits is also consistent with lack of gross anatomic deficits in children with mild HIE $[4,62]$. This model did not, however, demonstrate significant deficits in motor learning or visual-spatial learning that would more directly correlate to cognitive deficits sometimes seen in these children [7]. Together, these data suggest that this model allows for understanding the contribution of prenatal injury to long-term outcomes in the absence of significant structural injury.

The significance of this model stems from its relationship to the clinically-relevant context of mild HIE and has some features that can complement gaps in the well-established postnatal models $[15,16]$. One of the strengths of this model is that like most HIE in neonates, it is a prenatal injury and, therefore, can be used to understanding the physiology of in utero insult. Understanding the specific effects of pathologic hypoxia in the in utero environment is important because the fetal environment is intrinsically hypoxic at baseline $[51,27]$. The compensation for further hypoxia may be different in the fetal brain compared to the postnatal brain considering that the fetal brain is already more likely to use anaerobic respiration than the postnatal brain for energy [63]. Hif1 $\alpha$ can regulate metabolism [64] and in neural cells is required for normal brain development [65], indicating the importance of hypoxia for cell survival and differentiation. In this study, we corroborated previous findings that Hif1 $\alpha$ protein levels are stabilized in the fetal cortex of normoxic animals [51]. We further demonstrated an increase within the first 4 hours of hypoxia that was correlated to induction of one of its downstream targets, Vegfa. Interestingly, Hif1 $\alpha$ levels peak at 4 hours of hypoxia and then decreases after 8 hours. This decrease suggests that there may be an initial capacity to compensate for hypoxia that is lost over time. The fluctuation of HIF1 $\alpha$ levels during different duration of hypoxia may someday be found to have clinical relevance since the duration of in utero hypoxic injury prior to HIE can be elusive because frequently the diagnosis of HIE relies on recognition of the hypoxic event by mother or providers $[15,66]$.

Another advantage of a prenatal model is that it would allow more direct study of the effects of different pathologies of the maternal-placental unit on the pathophysiology of HIE. Conditions that 
lead to chronic prenatal hypoxia, such as excessive weight gain during pregnancy, are risk factors for worse outcomes from HIE [67]. This possible detrimental effect of prenatal chronic hypoxia on acute hypoxic neonatal injury is in sharp contrast to postnatal adult stroke studies were hypoxic preconditioning is protective of acute and long-term injury [68]. The epidemiological link between chronic hypoxia conditions in utero and acute hypoxic in utero injury warrants further study and this model is poised to study these acute-on-chronic interactions in a manner that cannot be directly studied in postnatal models.

This model may also be useful to study the effects of sex on outcomes from prenatal hypoxia by comparing these findings to other preclinical models. Clinical studies of long-term outcomes of HIE are underpowered to determine sex-related differences in long-term outcomes $[69,61]$ but have been suspected since there are sex differences from other causes of perinatal brain injury [70]. Sex differences have been observed in other models of hypoxia and hypoxic-ischemic injury $[71,70,72$, 49]. It is not clear if there are consistent sex-dichotomies in anxiety or autism in children after HIE. In particular, it is also not clear if the mild differences in females in social interactions in this mouse model has a true clinical correlate since males are typically most affected by autism in general [73]. There are sex-dependent regional differences in brain development[74]. The MRI studies here are underpowered to determine if hypoxia has sex-dichotomous effects on specific regions, but future studies in this model can focus on the possible relationship between specific regions and sexdichotomous behavioral differences.

In trying to determine the caveats and limitations of interpreting findings from this model, it is important to consider whether prenatal hypoxia models human "term" or "preterm" injury. The rodent brain at postnatal day 0 is similar to a 23-30 week gestation human brain and is considered human "term" equivalent at postnatal day 7-10 [17]. This prenatal model might be limited for studying some of the neuroanatomic effects of hypoxia relatively to term infants. However, it is suitable for studying the effects of hypoxia on acquisition of neurodevelopment milestones and functional networks in rodents that are equivalent between postnatal day 0 and term infants [21]. Mice do not have respiratory distress or feeding dyscoordination at birth that is frequently present in children born at less than 34 weeks gestation [22]. Furthermore, mice acquire the ability to crawl within a few days of life and are generally capable of immature walking by postnatal day 10 , which is generally a developmental milestone acquired in a 12 month old infant [21]. Thus, for future directions this model of prenatal hypoxia is well-suited to study the effect of hypoxia on these clinically relevant networks that are still developing in the term human brain through behavior and electrophysiology. Additionally, this model is likely more suitable for studying the neuroanatomic effects of hypoxia in preterm HIE, which is an understudied but likely important clinical modulator of 
neurodevelopmental outcomes in preterm infants [75]. While white matter microstructure is affected in premature infants and we did not see differences in the selected ROIs of corpus callosum (e.g., genu or splenium), lack of abnormality in the corpus callosum does not rule out more subtle effects in white matter development [76]. Future ex vivo MRI studies with larger sample sizes may help to systematically quantify the potential subtle difference of white matter microstructure between mice exposed to normoxia or hypoxia prenatally. Similarly, there may also be more subtle differences in neuron structure that are not captured by gray matter thickness or increase in ex vacuo ventriculomegaly.

Another limitation of this model includes that it does not have a true "ischemic" component and it lacks gross neuronal injury that has been associated with the most severe cases of children with HIE and the cell death seen in the Vannucci model [18]. Animals in the Vannucci model demonstrate visual spatial learning deficits [16] that are absent in this model; it is likely these deficits in the Vannucci model are related to the extent of hippocampal cell death and injury from the carotid ligation and not to the differences in gestational age. The need for both hypoxia and ischemia for significant cell death in vivo is supported in prenatal sheep studies that directly compare transient hypoxia to transient hypoxia-ischemia [29, 30]. These differences in cell survival between hypoxia only and hypoxia with ischemia are replicated in cell culture; neurons can survive in hypoxia alone but are much more susceptible to cell death in the setting of hypoxia with glucose depletion [77]. It is, thus, possible that in prenatal hypoxia the absence of cell death and absence of subsequent longterm anatomic injury is due to the absence of ischemia. Like transient prenatal hypoxia in sheep, though, we demonstrate long-term functional deficits, most notably in seizure threshold differences. Therefore, this model can be leveraged to understand the molecular mechanisms driven primarily by hypoxia that lead to functional deficits.

In conclusion, here we present a simplified, easy to reproduce, model of prenatal hypoxic injury that phenocopies many of the functional deficits consistent with findings in children with mild HIE. We expect these results will provide a platform to study the effects of prenatal risk factors and sex-dichotomous effects of hypoxia. This prenatal model may also provide an attractive platform for studying the genetic factors that exacerbate the mild phenotype (e.g. determine which factors protect from cell death in the setting of hypoxia only) and thereby provide mechanistic insight into why $40 \%$ of patients have moderate to severe disability after HIE. Ultimately, with comparative

509 studies amongst models of prenatal and postnatal injury we can better understand the full spectrum

510 of relevant disease from hypoxia to the developing brain and determine opportunities for novel 511 interventions. 


\section{Acknowledgements}

514 We thank the Small Animal Imaging Facility at the Research Institute of the Children's Hospital of

515 Philadelphia (CHOP), the CHOP Pathology Core, and the Neurobehavior Testing Core, and

516 EEG/Epilepsy core at UPenn and IDDRC at CHOP/Penn [Grant: U54 HD086984] for assistance. We

517 thank Dr. Rui Xiao in the Department of Biostatistics at UPenn for her advice on statistical analysis.

518 We thank Brenna Daugherty for initial studies monitoring pregnant mouse locomotion. We are

519 grateful to Daniel Licht and the Wolfson family for providing the Biospherix oxygen chambers. We are

520 grateful to Dr. Michal Elovitz and members of the Marsh and Anderson labs for numerous insightful

521 discussions conversations regarding the data in this manuscript.

522

523

\section{Statement of Ethics}

524 Studies involving animals were approved by the Institutional Animal Care and Use Committee

525 (IACUC) at the Children's Hospital of Philadelphia.

526

\section{Conflict of Interest Statement}

528 The authors have no conflicts of interest to declare.

Funding Sources

531 This work was supported by the National Institutes of Health [Grant: 5K12HD043245-18,

532 R01MH092535, R01MH092535-S1 and U54HD086984], and institutional grants from the Children's

533 Hospital of Philadelphia, including Neurology Black Tie Tailgate Fund, Foerderer Grant, and K-

534 readiness Pilot award.

\section{Author Contributions}

537 A.G.C conceived the project and designed, performed, analyzed and interpreted components of all

538 the experiments. E.C.G. completed immunoblots and behavior experiments. I.M.S. completed

539 behavior experiments and assessed maternal dam health. C.Z and S.M. performed the MRI

540 experiments. M.O. and H.H. analyzed the MRI experiments. A.N.V. completed the histology for

541 apoptosis. S.A.A. and E.D.M. participated in conceiving the project and interpretation of the data. 
bioRxiv preprint doi: https://doi.org/10.1101/2021.08.04.451528; this version posted September 30, 2021. The copyright holder for this preprint (which was not certified by peer review) is the author/funder. All rights reserved. No reuse allowed without permission.

542 A.G.C wrote the initial manuscript and finalized all versions. All other authors participated in revisions

543 of the manuscript.

544

545 Data Availability Statement

546 All data generated or analyzed during this study are included in this article. Further enquiries can be

547 directed to the corresponding author. 


\section{References}

1. Lee AC, Kozuki N, Blencowe H, Vos T, Bahalim A, Darmstadt GL, et al. Intrapartum-related neonatal encephalopathy incidence and impairment at regional and global levels for 2010 with trends from 1990. Pediatr Res. 2013 Dec;74 Suppl 1(SUPPL. 1):50-72.

2. Stanaway JD, Afshin A, Gakidou E, Lim SS, Abate D, Abate KH, et al. Global, regional, and national comparative risk assessment of 84 behavioural, environmental and occupational, and metabolic risks or clusters of risks for 195 countries and territories, 1990-2017: a systematic analysis for the Global Burden of Disease Study 2017. The Lancet. 2018;392(10159):1923-94.

3. de Haan M, Wyatt JS, Roth S, Vargha-Khadem F, Gadian D, Mishkin M. Brain and cognitivebehavioural development after asphyxia at term birth. Dev Sci. 2006 Jul;9(4):350-8.

4. van Kooij BJ, van Handel M, Nievelstein RA, Groenendaal F, Jongmans MJ, de Vries LS. Serial $\mathrm{MRI}$ and neurodevelopmental outcome in 9- to 10-year-old children with neonatal encephalopathy. J Pediatr. 2010 Aug;157(2):221-27 e2.

5. Eunson P. The long-term health, social, and financial burden of hypoxic-ischaemic encephalopathy. Dev Med Child Neurol. 2015 Apr;57 Suppl 3:48-50.

6. Reiss J, Sinha M, Gold J, Bykowski J, Lawrence SM. Outcomes of Infants with Mild Hypoxic Ischemic Encephalopathy Who Did Not Receive Therapeutic Hypothermia. Biomed Hub. 2019 Sep-Dec;4(3):1-9.

7. Finder M, Boylan GB, Twomey D, Ahearne C, Murray DM, Hallberg B. Two-Year Neurodevelopmental Outcomes After Mild Hypoxic Ischemic Encephalopathy in the Era of Therapeutic Hypothermia. JAMA Pediatr. 2020 Jan 1;174(1):48-55.

8. SchregImann M, Ground A, Vollmer B, Johnson MJ. Systematic review: long-term cognitive and behavioural outcomes of neonatal hypoxic-ischaemic encephalopathy in children without cerebral palsy. Acta Paediatr. 2020 Jan;109(1):20-30.

9. Ahearne $\mathrm{CE}$, Boylan GB, Murray DM. Short and long term prognosis in perinatal asphyxia: An update. World J Clin Pediatr. 2016 Feb 8;5(1):67-74.

10. Chin EM, Jayakumar S, Ramos E, Gerner G, Soares BP, Cristofalo E, et al. Preschool Language Outcomes following Perinatal Hypoxic-Ischemic Encephalopathy in the Age of Therapeutic Hypothermia. Dev Neurosci. 2019 Jun 5:1-11.

11. Hutter D, Kingdom J, Jaeggi E. Causes and mechanisms of intrauterine hypoxia and its impact on the fetal cardiovascular system: a review. Int J Pediatr. 2010;2010:401323.

12. Jauniaux E, Watson AL, Hempstock J, Bao YP, Skepper JN, Burton GJ. Onset of maternal arterial blood flow and placental oxidative stress. A possible factor in human early pregnancy failure. Am J Pathol. 2000 Dec;157(6):2111-22.

13. Panfoli I, Candiano G, Malova M, De Angelis L, Cardiello V, Buonocore G, et al. Oxidative Stress as a Primary Risk Factor for Brain Damage in Preterm Newborns. Front Pediatr. 2018;6:369.

14. Rice JE, 3rd, Vannucci RC, Brierley JB. The influence of immaturity on hypoxic-ischemic brain damage in the rat. Ann Neurol. 1981 Feb;9(2):131-41.

15. Ferriero DM. Neonatal brain injury. N Engl J Med. 2004 Nov 4;351(19):1985-95.

16. Sun H, Juul HM, Jensen FE. Models of hypoxia and ischemia-induced seizures. J Neurosci Methods. 2016 Feb 15;260:252-60.

17. Semple BD, Blomgren K, Gimlin K, Ferriero DM, Noble-Haeusslein LJ. Brain development in rodents and humans: Identifying benchmarks of maturation and vulnerability to injury across species. Pergamon; 2013. p. 1-16.

18. Northington FJ, Chavez-Valdez R, Martin LJ. Neuronal cell death in neonatal hypoxiaischemia. Ann Neurol. 2011 May;69(5):743-58.

19. Badawi N, Kurinczuk JJ, Keogh JM, Alessandri LM, O'Sullivan F, Burton PR, et al. Antepartum 
risk factors for newborn encephalopathy: the Western Australian case-control study. BMJ. 1998 Dec 5;317(7172):1549-53.

20. Kurinczuk JJ, White-Koning M, Badawi N. Epidemiology of neonatal encephalopathy and hypoxic-ischaemic encephalopathy. Elsevier; 2010. p. 329-38.

21. Feather-Schussler DN, Ferguson TS. A Battery of Motor Tests in a Neonatal Mouse Model of Cerebral Palsy. J Vis Exp. 2016 Nov 3(117):e53569-e69.

22. Behrman RE, Butler AS. Mortality and acute complications in preterm infants. Preterm birth: causes, consequences \& prevention. 2006.

23. Aziz NM, Guedj F, Pennings JLA, Olmos-Serrano JL, Siegel A, Haydar TF, et al. Lifespan analysis of brain development, gene expression and behavioral phenotypes in the Ts1Cje, Ts65Dn and Dp(16)1/Yey mouse models of Down syndrome. Dis Model Mech. 2018 Jun $12 ; 11(6)$.

24. Baud O, Daire JL, Dalmaz Y, Fontaine RH, Krueger RC, Sebag G, et al. Gestational hypoxia induces white matter damage in neonatal rats: a new model of periventricular leukomalacia. Brain Pathol. 2004 Jan;14(1):1-10.

25. Mallard C, Vexler ZS. Modeling ischemia in the immature brain: How translational are animal models? 2015.

26. Kubo KI, Deguchi K, Nagai T, Ito Y, Yoshida K, Endo T, et al. Association of impaired neuronal migration with cognitive deficits in extremely preterm infants. JCI Insight. 2017 May 18;2(10).

27. Howell KR, Pillai A. Effects of prenatal hypoxia on schizophrenia-related phenotypes in heterozygous reeler mice: a gene $x$ environment interaction study. Eur Neuropsychopharmacol. 2014 Aug;24(8):1324-36.

28. Howell KR, Pillai A. Long-Term Effects of Prenatal Hypoxia on Schizophrenia-Like Phenotype in Heterozygous Reeler Mice. Mol Neurobiol. 2016 Jul;53(5):3267-76.

29. McClendon E, Shaver DC, Degener-O'Brien K, Gong X, Nguyen T, Hoerder-Suabedissen A, et al. Transient Hypoxemia Chronically Disrupts Maturation of Preterm Fetal Ovine Subplate Neuron Arborization and Activity. J Neurosci. 2017 Dec 6;37(49):11912-29.

30. McClendon E, Wang K, Degener-O'Brien K, Hagen MW, Gong X, Nguyen T, et al. Transient Hypoxemia Disrupts Anatomical and Functional Maturation of Preterm Fetal Ovine CA1 Pyramidal Neurons. J Neurosci. 2019 Oct 2;39(40):7853-71.

31. Deacon RM. Assessing nest building in mice. Nat Protoc. 2006;1(3):1117-9.

32. Huang $\mathrm{H}$, Zhang J, Wakana S, Zhang W, Ren T, Richards LJ, et al. White and gray matter development in human fetal, newborn and pediatric brains. Neuroimage. 2006 Oct 15;33(1):27-38.

33. Huang $\mathrm{H}$, Yamamoto A, Hossain MA, Younes L, Mori S. Quantitative cortical mapping of fractional anisotropy in developing rat brains. J Neurosci. 2008 Feb 6;28(6):1427-33.

34. Huang $\mathrm{H}$, Xue R, Zhang J, Ren T, Richards LJ, Yarowsky P, et al. Anatomical characterization of human fetal brain development with diffusion tensor magnetic resonance imaging. J Neurosci. 2009 Apr 1;29(13):4263-73.

35. Jiang H, van Zijl PC, Kim J, Pearlson GD, Mori S. DtiStudio: resource program for diffusion tensor computation and fiber bundle tracking. Comput Methods Programs Biomed. 2006 Feb;81(2):106-16.

36. Paxinos G, Franklin KBJ. Paxinos and Franklin's the Mouse Brain in Stereotaxic Coordinates. 2001.

37. Deacon RM. Digging and marble burying in mice: simple methods for in vivo identification of biological impacts. Nat Protoc. 2006;1(1):122-4.

38. Vorhees CV, Williams MT. Morris water maze: procedures for assessing spatial and related forms of learning and memory. Nat Protoc. 2006;1(2):848-58.

39. Witkin JM. Animal Models of Obsessive-Compulsive Disorder. Hoboken, NJ, USA: John Wiley 
\& Sons, Inc.; 2008. p. 9.30.1-9.30.9.

40. Yang M, Silverman JL, Crawley JN. Automated three-chambered social approach task for mice. Curr Protoc Neurosci. 2011 Jul;Chapter 8:Unit 826.

41. Rothwell PE, Fuccillo MV, Maxeiner S, Hayton SJ, Gokce O, Lim BK, et al. Autism-associated neuroligin-3 mutations commonly impair striatal circuits to boost repetitive behaviors. Cell. 2014 Jul 3;158(1):198-212.

42. Simonet JC, Sunnen CN, Wu J, Golden JA, Marsh ED. Conditional Loss of Arx From the Developing Dorsal Telencephalon Results in Behavioral Phenotypes Resembling Mild Human ARX Mutations. Cereb Cortex. 2015 Sep;25(9):2939-50.

43. Tucker LB, McCabe JT. Behavior of Male and Female C57BL/6J Mice Is More Consistent with Repeated Trials in the Elevated Zero Maze than in the Elevated Plus Maze. Front Behav Neurosci. 2017;11:13.

44. Kadiyala SB, Papandrea D, Herron BJ, Ferland RJ. Segregation of seizure traits in C57 black mouse substrains using the repeated-flurothyl model. PLoS One. 2014;9(3):e90506.

45. Ferland RJ. The Repeated Flurothyl Seizure Model in Mice. Bio Protoc. 2017 Jun 5;7(11).

46. Halekoh U, Højsgaard S, Yan J. TheRPackagegeepackfor Generalized Estimating Equations. Journal of Statistical Software. 2006;15(2):1-11.

47. Bates D, Mächler M, Bolker B, Walker S. Fitting Linear Mixed-Effects Models Usinglme4. Journal of Statistical Software. 2015;67(1).

48. Demarest TG, Schuh RA, Waddell J, McKenna MC, Fiskum G. Sex-dependent mitochondrial respiratory impairment and oxidative stress in a rat model of neonatal hypoxic-ischemic encephalopathy. J Neurochem. 2016 Jun;137(5):714-29.

49. Aravamuthan BR, Gandham S, Young AB, Rutkove SB. Sex may influence motor phenotype in a novel rodent model of cerebral palsy. Neurobiol Dis. 2020 Feb;134:104711.

50. Semenza GL. Oxygen sensing, hypoxia-inducible factors, and disease pathophysiology. Annu Rev Pathol. 2014;9(1):47-71.

51. Trollmann R, Strasser K, Keller S, Antoniou X, Grenacher B, Ogunshola OO, et al. Placental HIFs as markers of cerebral hypoxic distress in fetal mice. Am J Physiol Regul Integr Comp Physiol. 2008 Dec;295(6):R1973-81.

52. Nakajima W, Ishida A, Lange MS, Gabrielson KL, Wilson MA, Martin LJ, et al. Apoptosis has a prolonged role in the neurodegeneration after hypoxic ischemia in the newborn rat. J Neurosci. 2000 Nov 1;20(21):7994-8004.

53. Belet N, Belet U, Incesu L, Uysal S, Ozinal S, Keskin T, et al. Hypoxic-ischemic encephalopathy: correlation of serial MRI and outcome. Pediatr Neurol. 2004 Oct;31(4):26774.

54. Brooks SP, Dunnett SB. Tests to assess motor phenotype in mice: A user's guide. 2009.

55. Jaramillo TC, Speed HE, Xuan Z, Reimers JM, Escamilla CO, Weaver TP, et al. Novel Shank3 mutant exhibits behaviors with face validity for autism and altered striatal and hippocampal function. Autism Res. 2017 Jan;10(1):42-65.

56. Drapeau E, Riad M, Kajiwara Y, Buxbaum JD. Behavioral Phenotyping of an Improved Mouse Model of Phelan-McDermid Syndrome with a Complete Deletion of the Shank3 Gene. eNeuro. 2018 May-Jun;5(3).

57. Cryan JF, Holmes A. Model organisms: The ascent of mouse: Advances in modelling human depression and anxiety. 2005.

58. Van Handel M, Swaab H, De Vries LS, Jongmans MJ. Long-term cognitive and behavioral consequences of neonatal encephalopathy following perinatal asphyxia: A review. 2007.

59. van Handel M, Swaab H, de Vries LS, Jongmans MJ. Behavioral outcome in children with a history of neonatal encephalopathy following perinatal asphyxia. J Pediatr Psychol. 2010 Apr;35(3):286-95.

60. Barnett A, Mercuri E, Rutherford M, Haataja L, Frisone MF, Henderson S, et al. Neurological 
and perceptual-motor outcome at 5 - 6 years of age in children with neonatal encephalopathy: relationship with neonatal brain MRI. Neuropediatrics. 2002 Oct;33(5):2428.

61. Shankaran S, Pappas A, McDonald SA, Vohr BR, Hintz SR, Yolton K, et al. Childhood outcomes after hypothermia for neonatal encephalopathy. N Engl J Med. 2012 May 31;366(22):208592.

62. Annink KV, de Vries LS, Groenendaal F, van den Heuvel MP, van Haren NEM, Swaab H, et al. The long-term effect of perinatal asphyxia on hippocampal volumes. Pediatr Res. 2019 Jan;85(1):43-49.

63. Oyarzábal A, Musokhranova U, Barros L, García-Cazorla A. Energy metabolism in childhood neurodevelopmental disorders. EBioMedicine. 2021 Jul;69:103474.

64. Gaspar JM, Velloso LA. Hypoxia Inducible Factor as a Central Regulator of Metabolism Implications for the Development of Obesity. Front Neurosci. 2018 11/01/2018;12:813.

65. Tomita S, Ueno M, Sakamoto M, Kitahama Y, Ueki M, Maekawa N, et al. Defective brain development in mice lacking the Hif-1alpha gene in neural cells. Mol Cell Biol. 2003 Oct;23(19):6739-49.

66. Perlman JM. Pathogenesis of hypoxic-ischemic brain injury. Journal of Perinatology. 2007;27(S1):S39-S46.

67. Peebles PJ, Duello TM, Eickhoff JC, McAdams RM. Antenatal and intrapartum risk factors for neonatal hypoxic ischemic encephalopathy. J Perinatol. 2020 Jan;40(1):63-69.

68. Stowe AM, Altay T, Freie AB, Gidday JM. Repetitive hypoxia extends endogenous neurovascular protection for stroke. Ann Neurol. 2011 Jun;69(6):975-85.

69. Glass HC, Hong KJ, Rogers EE, Jeremy RJ, Bonifacio SL, Sullivan JE, et al. Risk factors for epilepsy in children with neonatal encephalopathy. Pediatr Res. 2011 Nov;70(5):535-40.

70. Smith AL, Alexander M, Rosenkrantz TS, Sadek ML, Fitch RH. Sex differences in behavioral outcome following neonatal hypoxia ischemia: insights from a clinical meta-analysis and a rodent model of induced hypoxic ischemic brain injury. Exp Neurol. 2014 Apr;254:54-67.

71. Hill CA, Fitch RH. Sex differences in mechanisms and outcome of neonatal hypoxia-ischemia in rodent models: Implications for sex-specific neuroprotection in clinical neonatal practice. 2012.

72. Charriaut-Marlangue C, Besson VC, Baud O. Sexually dimorphic outcomes after neonatal stroke and hypoxia-ischemia. MDPI AG; 2018.

73. Baron-Cohen S, Knickmeyer RC, Belmonte MK. Sex differences in the brain: implications for explaining autism. Science. 2005 Nov 4;310(5749):819-23.

74. Qiu LR, Fernandes DJ, Szulc-Lerch KU, Dazai J, Nieman BJ, Turnbull DH, et al. Mouse MRI shows brain areas relatively larger in males emerge before those larger in females. Nat Commun. 2018 Jul 5;9(1):2615.

75. Gopagondanahalli KR, Li J, Fahey MC, Hunt RW, Jenkin G, Miller SL, et al. Preterm hypoxicischemic encephalopathy. Frontiers Media SA; 2016. p. 114-14.

76. Salmaso N, Jablonska B, Scafidi J, Vaccarino FM, Gallo V. Neurobiology of premature brain injury. Nat Neurosci. 2014 Mar;17(3):341-6.

77. Almeida A, Delgado-Esteban M, Bolanos JP, Medina JM. Oxygen and glucose deprivation induces mitochondrial dysfunction and oxidative stress in neurones but not in astrocytes in primary culture. J Neurochem. 2002 Apr;81(2):207-17. 


\section{Figure Legends}

Fig. 1: Prenatal hypoxia paradigm induces a canonical HIF1 $\alpha$ response consistent with hypoxic insult in the fetal brains. (A) Representative immunoblot if HIF1 $\alpha$ protein in fetal brains after indicated time of prenatal hypoxia. (B) HIF1 $\alpha$ protein quantification shown. Statistics by One-Way ANOVA with Dunnett's multiple comparison test for statistical significance. (C) Vegfa mRNA levels after time of prenatal hypoxia. Points shown in graphs are from individual fetal brains. Nested OneWay ANOVA with Dunnett's multiple comparison test for statistical significance.

Fig. 2: Maternal dams make normal nests and there is no early difference in litter health. $(A)$ Cumulative distance over time of pregnant dams during the first 90 minutes the mice were in chamber. Area under the curve is shaded and the dashed line around each condition represents SEM of respective condition. Dashed black line is where mice reach $10 \% 02$. Statistics shown for the first 20 minutes of chamber exposure and then for 1 hour after hypoxic mice reached goal oxygen level. (B) Scoring of maternal nestlets the morning after normoxia or hypoxia. Points represent individual dams that were assessed. (C) Litter size at birth and (D) gestational age at birth for indicated individual litters were assessed. Welch's t-test used for (A-D). (E) Percent survival of offspring. Error bars represent standard error at that time point. Log-rank rest used for statistics between normoxia (solid black line) and hypoxia (dashed blue line).

Fig. 3: Prenatal hypoxia does not increase cell death in the fetal brain. (A) Representative image of apoptotic nuclei (arrows) within cortex, hematoxylin and eosin stain. Scale bar represents $50 \mu \mathrm{M}$ (BD) Number of apoptotic nuclei in in the cortex, basal ganglia, and white matter, reported as average seen in 10 separate high-powered fields. Welch's t-test was used to determine statistical significance.

Fig. 4: Prenatal hypoxia does not result in long-term gross neuroanatomical damage (A) Axial images of adult female brain MRI (averaged b0 map) of indicated conditions from individual mice. (B) Quantification of ventricular size in the setting of normoxia and hypoxia. Hypoxic animals from (A) represented by indicated symbols in (B). (C) Mid-sagittal image demonstrating the areas that were quantified, the anterior cingulate cortex (light blue line) the genu of the corpus callosum (orange circle), and the splenium of the corpus callosum (pink circle). (D) Quantification of cortical 
thickness at the anterior cingulate. (E \& F) Quantification of fractional anisotropy in mid-sagittal areas of the genu and splenium of the corpus callosum respectively. Statistics as outlined in the methods. Points represent individual mice.

Fig. 5: Prenatal hypoxia leads to functional deficits in adult mice. (A) Flurothyl seizure threshold study demonstrating time to first GTC. (B \& C) Data from Grip strength. (B) Forelimbs and (C) hindlimbs force. (E) Number of marbles buried. (F) ) Change in nestlet weight in short term nestlet test. Statistics as outlined in the methods. Points represent individual mice.

Fig. 6. Sex-dichotomous behaviour differences after prenatal hypoxia. (A) Time spent in open arms in Elevated zero maze. (B) Total time animals spent in the center of Open field. (A-D) Percent time in each individual chamber during stages of social interaction study. (A-B) Data from habituation stage from males and females. (C-D) Data from novel mouse/object stage from males and female. (E-H) Percent time mice spent sniffing at the plastic clear tube. Statistics as outlined in the methods. Points represent individual mice.

Fig. 7: Prenatal hypoxia does not lead to deficits in learning or memory. (A) Speed at fall on Rotarod. (B-C) Data from Morris Water Maze. (B) ) Latency time to platform in place trials. (C) Onehour probe trial. (D) Twenty-four hour probe trial. Statistics performed as detailed in methods. Each point represents a single animal. 
Figure 1

A

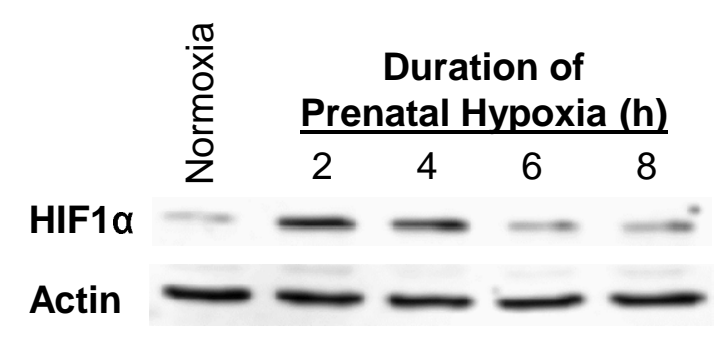

B

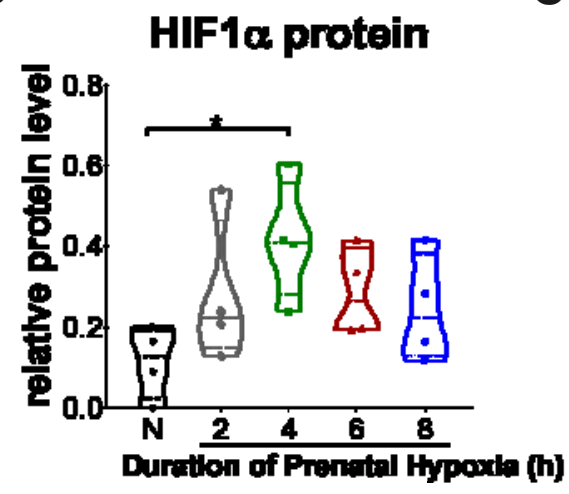

C

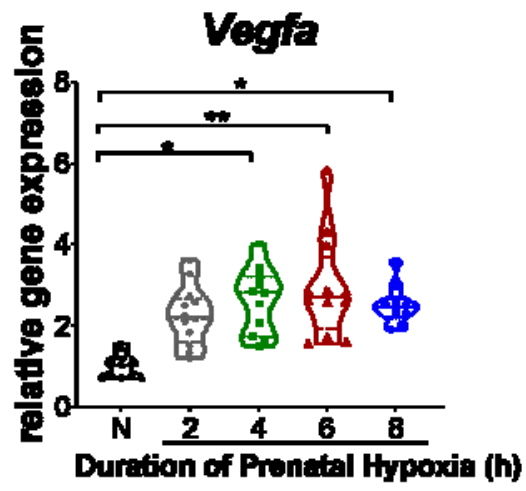


Figure 2

A

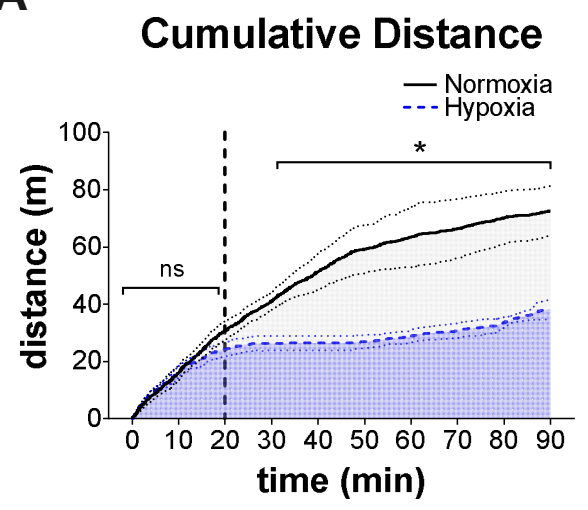

B Neslet Structure

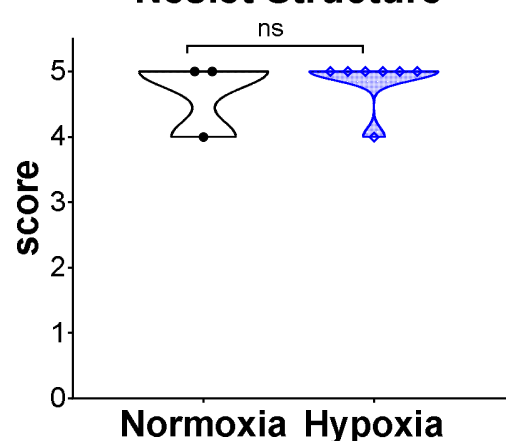

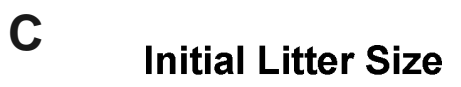

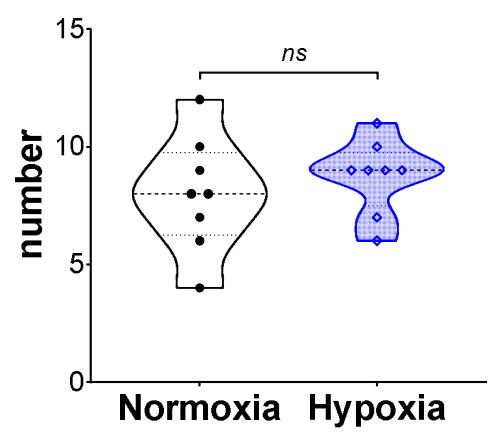

D Gestational Age at Birth

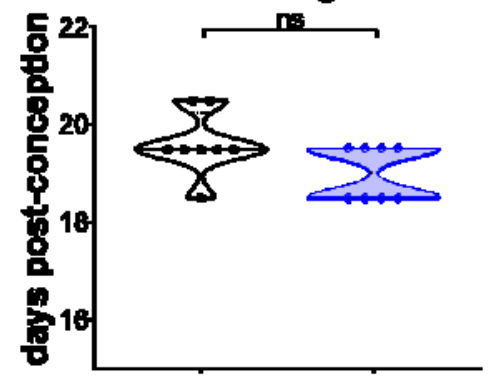

Normoxia Hypoxia
E Offspring Survival

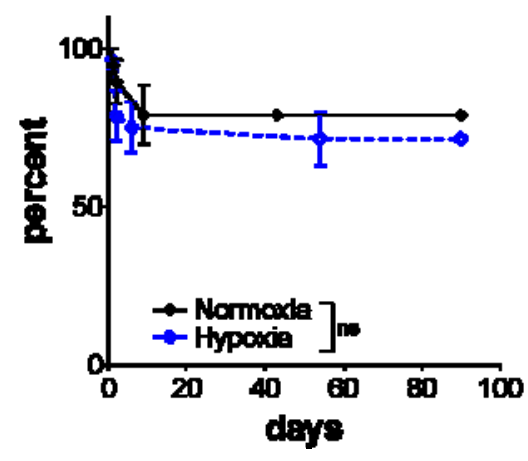




\section{Figure 3}

A

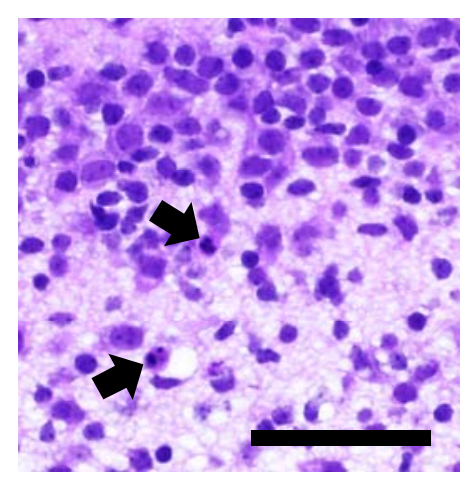

C

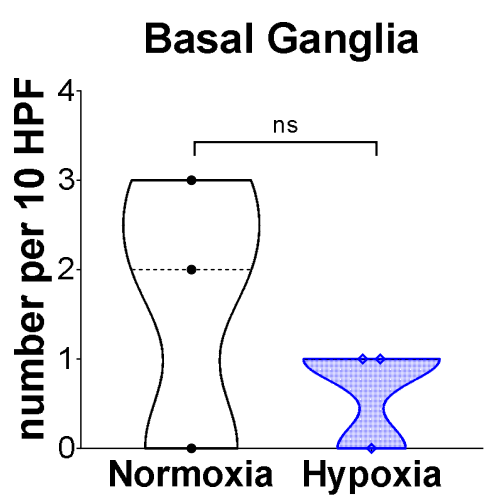

B

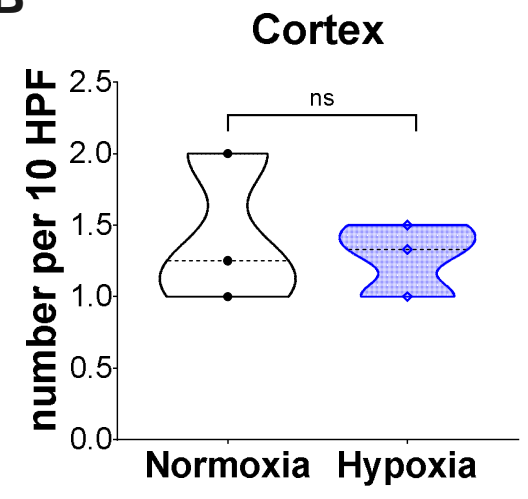

D

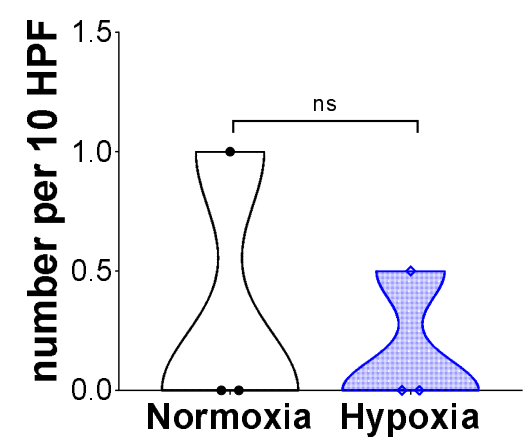




\section{Figure 4}

A

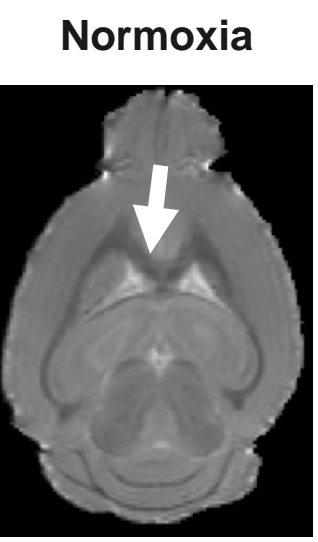

C

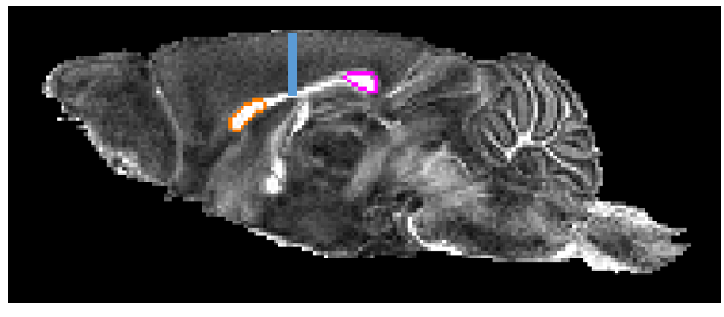
(Severely Affected)

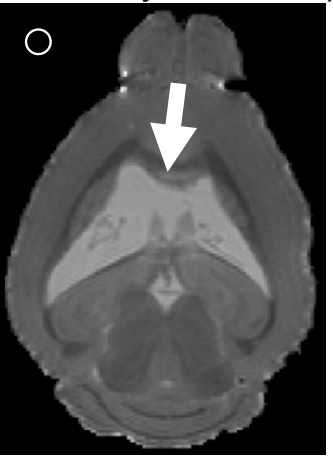

B

D

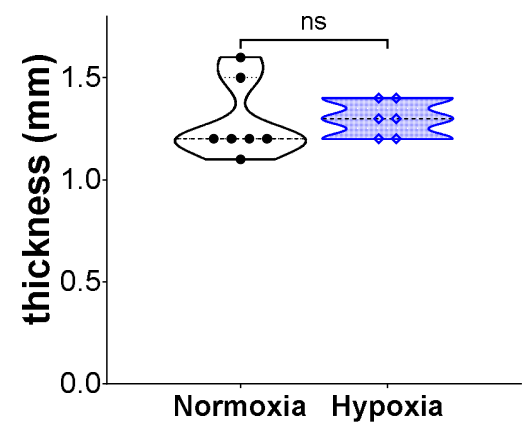

E

$F$
Splenium of the Corpus Callosum

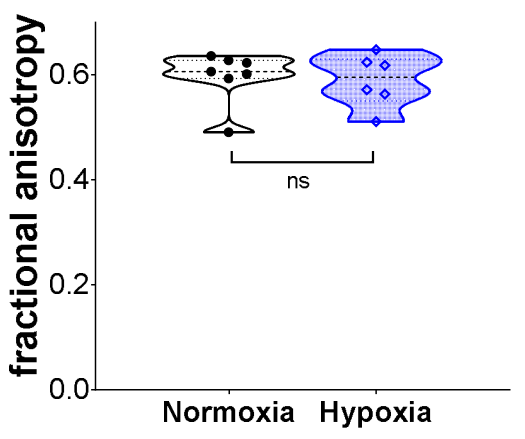

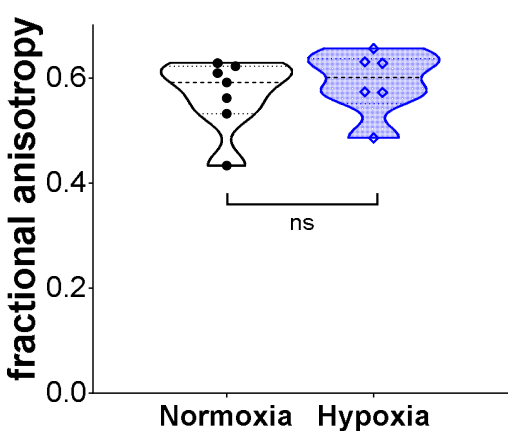




\section{Figure 5}
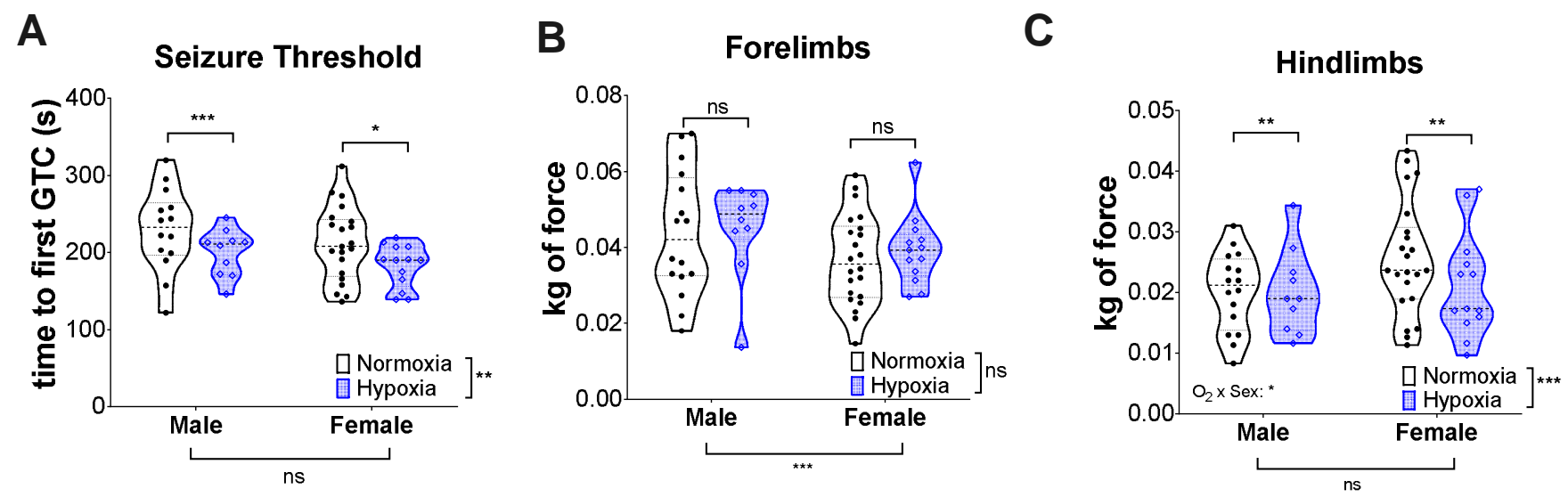

D

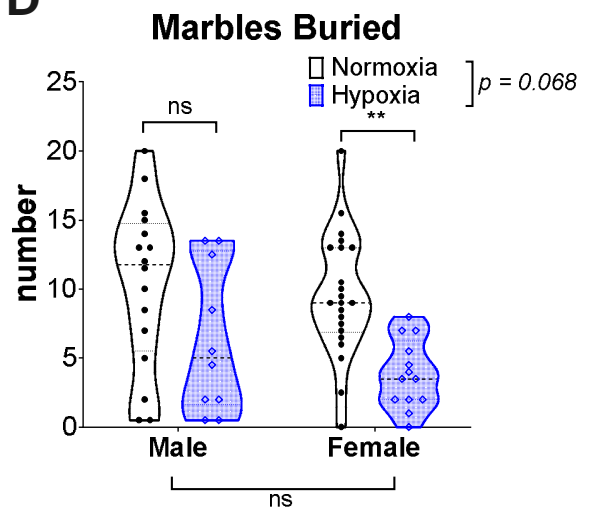

E Short Term Nestlet

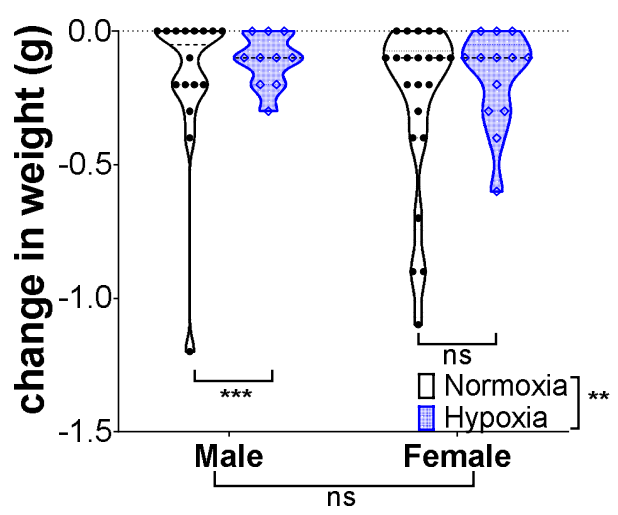




\section{Figure 6}

A

Time in Open Arm
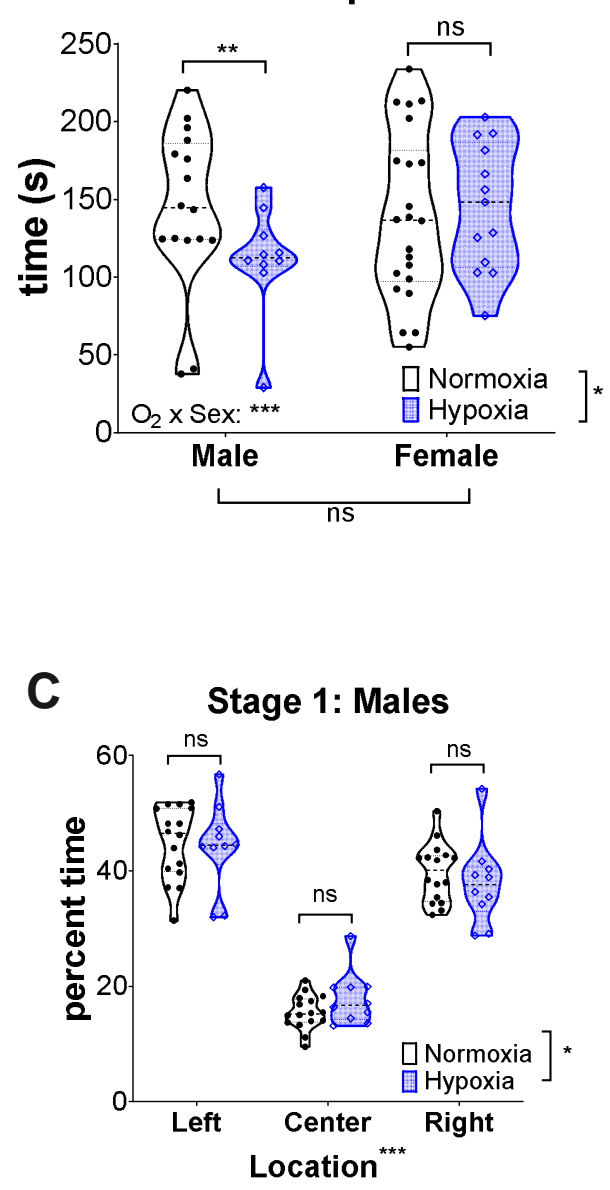

$\mathbf{E}$

Stage 2: Males

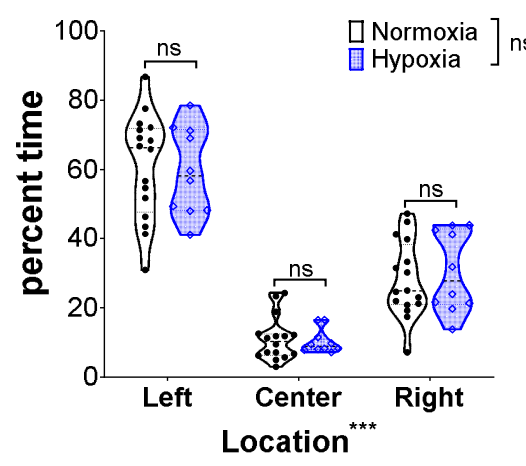

B Time in Open Center

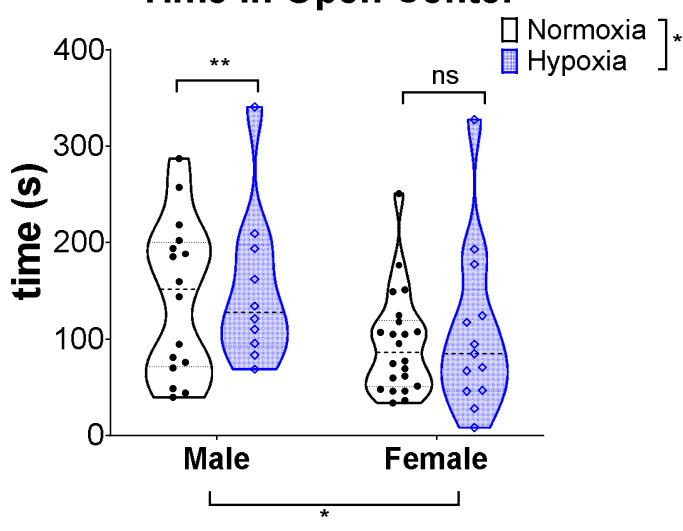

D

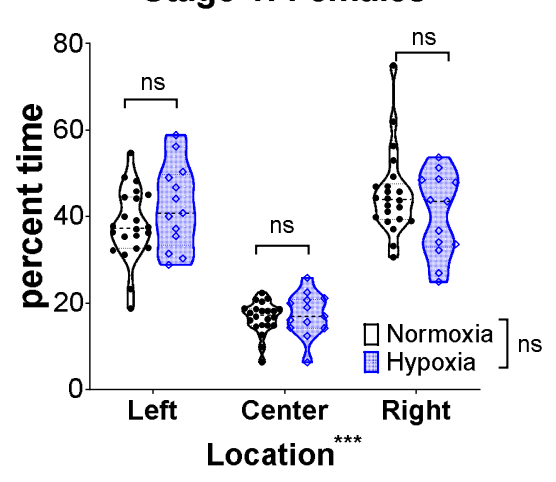

$\mathbf{F}$

Stage 2: Females

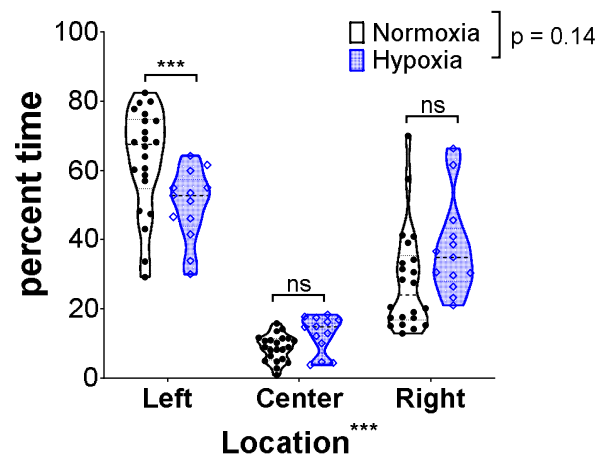




\section{Figure 7}

A

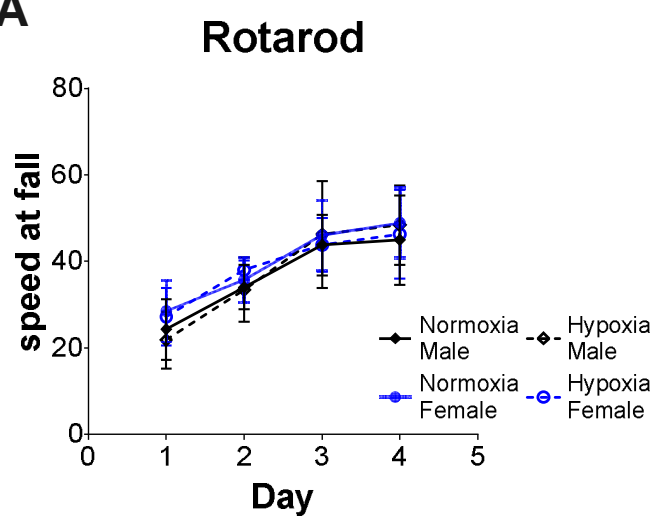

C

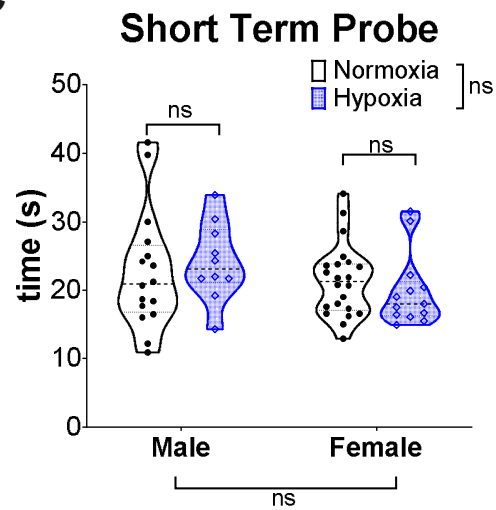

B

\section{Morris Water Maze}

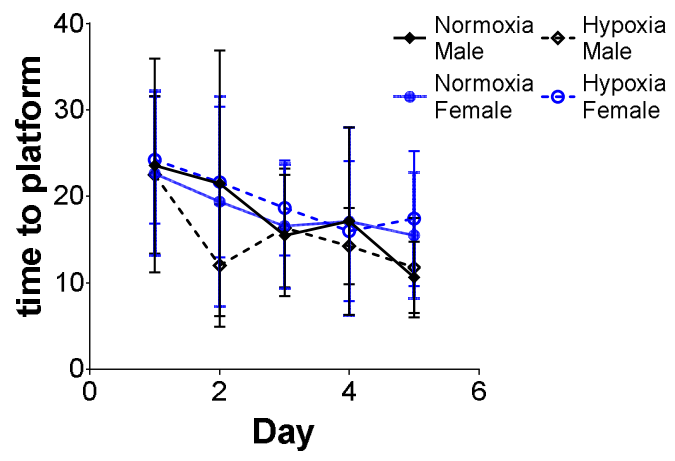

D

Long Term Probe

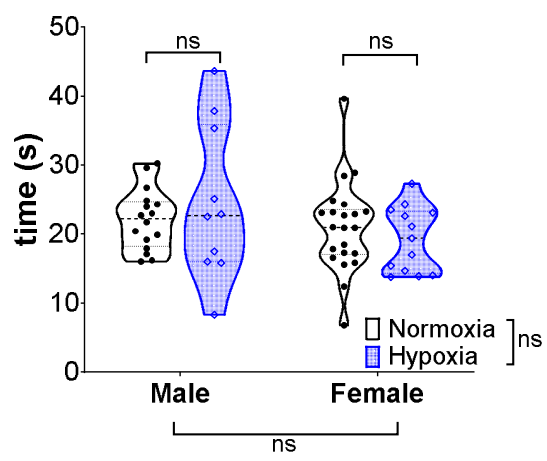

